\title{
Management of Giant Retinal Tear with microincision vitrectomy and metallic retinal tacks fixation-a case report
}

\author{
Yo-Chen Chang ${ }^{1,2}$, Li-Yi Chiu', Tzu-En Kao', Wen-Hsin Cheng ${ }^{1}$, Ting-An Chen ${ }^{1}$ and Wen-Chuan Wu ${ }^{1,2^{*}}$ (D)
}

\begin{abstract}
Background: Giant retinal tear is usually challenging among retinal detachment with recurrent rate up to $45 \%$. Here we presented a case of giant retinal tear being treated by microincision vitrectomy and retinal tacks fixation.

Case presentation: A 53-year-old male presented to our hospital with blurred vision of his right eye for one week with floaters and obscured sensation over nasal visual field. Ocular examination showed a 120 degree giant tear with large inverted flap and retinal detachment of his right eye. The BCVA was only naming digit. Under the impression of giant retinal tear with retinal detachment, 23-gauge pars plana vitrectomy were performed using Constellation high speed vitrectomy system and Topcon non-contact wide angle viewing system. During surgery, the vitreous was removed and perfluorocarbon liquids (PFCL) was injected to help unfolding the large inverted retinal flap. Three retinal tacks were applied to help fixating the large inverted retinal flap. Then, fluid-gas exchange, endolaser photocoagulation and intraocular silicone oil tamponade were performed as well. Initial reattachment of his right retina was achieved and his best corrected visual acuity improved to 0.3 of his right eye postoperatively. There was no recurrent retinal detachment during follow up period of 19 months.
\end{abstract}

Conclusions: Primary microincision vitrectomy using wide-angle viewing system with intraoperative perfluorocarbon liquids (PFCL) assistant, retinal tacks fixation and intraocular silicone oil tamponade appears to be safe and feasible for managing giant retinal tear with retinal detachment.

Keywords: Giant retinal tear, Microincision vitrectomy surgery, Retinal tack, Wide-angle viewing systems

\section{Background}

Giant retinal tear (GRT) is usually challenging among retinal detachment with recurrent rate up to $45 \%$ [1]. In order to manage such cases, the use of retinal tacks for the repair of complex retinal detachment such as GRT was introduced by Dr. Ando and Dr. de Juan in the 1980s [2, 3]. Microincisional vitrectomy surgery (MIVS) including 23-and 25-gauge was first reported in 2002 and 2005, which had significant reduction in conjunctival injection and postoperative pain and discomfort [4]. Although MIVS was widely used in managing retinal disease, there are no publications on GRT treated by MIVS and retinal tacks in Taiwan. Besides, Wide-angle

\footnotetext{
* Correspondence: wcwu.oph@gmail.com

${ }^{1}$ Department of Ophthalmology, Kaohsiung Medical University Hospital, Kaohsiung, Taiwan

${ }^{2}$ Department of Ophthalmology, School of Medicine, Kaohsiung Medical University, Kaohsiung, Taiwan
}

viewing systems (WAVs) offers a panoramic view in vitreoretinal surgery and it is extremely useful especially for giant retinal tear because the edges of retinal tear can be well seen in one view [5]. Therefore, the purpose of this case report was to present a case of GRT with RD treated by primary MIVS and retinal tacks with WAVs.

\section{Case presentation}

A 53-year-old male denied past medical and trauma history presented to our hospital with blurred vision of his right eye for one week with floaters and obscured sensation over nasal visual field. Ocular examination showed a 120 degree retinal tear (from 7 to 11 o'clock) with large inverted rigid flap and retinal detachment from 6 to 12 o'clock with macula-off in his right eye (Fig. 1a). The grading of proliferative vitreoretinopathy (PVR) was "Grade C". The BCVA of his right eye was only naming 


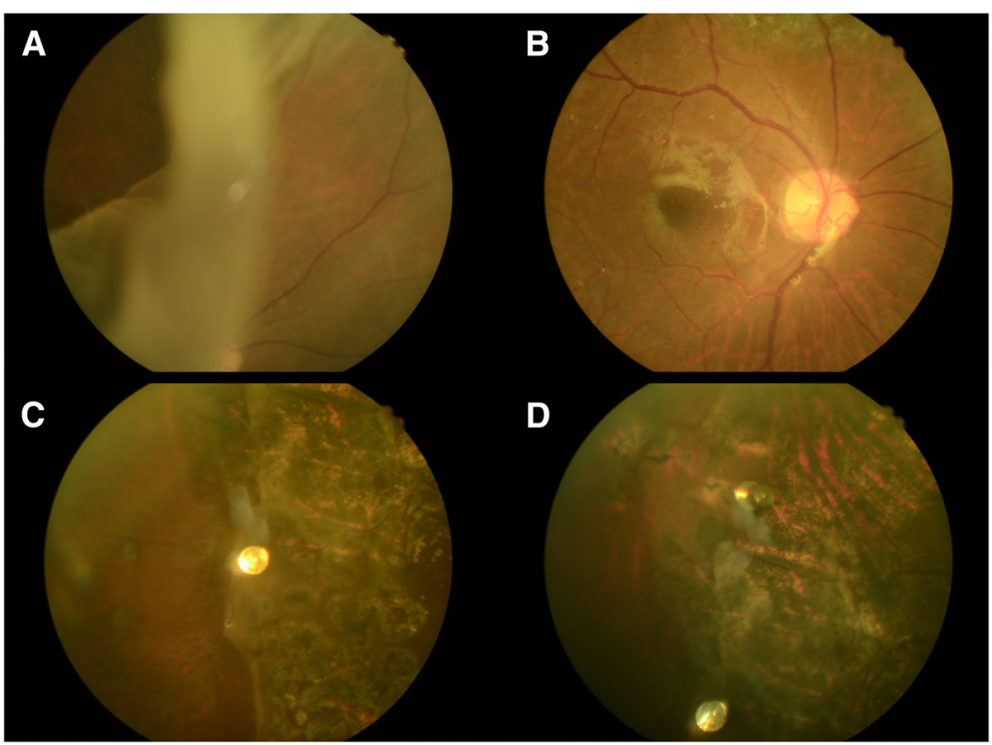

Fig. 1 a. Fundus examination revealed a 120 degree giant tear with large inverted flap of the 53-year-old man's right eye. b, c and d. No recurrent retinal detachment was noted during follow up period and the retina was well fixated by retinal tacks and laser scar

digit at his first presentation. Under the impression of GRT with retinal detachment, 23-gauge pars plana vitrectomy were performed using Constellation high speed vitrectomy system (Alcon Surgical, Fort Worth, TX, USA) and Topcon non-contact wide angle viewing system (Topcon Medical Inc. Livermore, CA, USA). During surgery, the temporal retina was noted to have a rolled and stiffened edge at the posterior aspect of the giant retinal tear (Fig. 2a). The vitreous was removed and tractions were relieved as much as possible, then perfluorocarbon liquids (PFCL) was injected to help unfolding the large inverted retinal flap (Fig. 2b). Three stainless steel

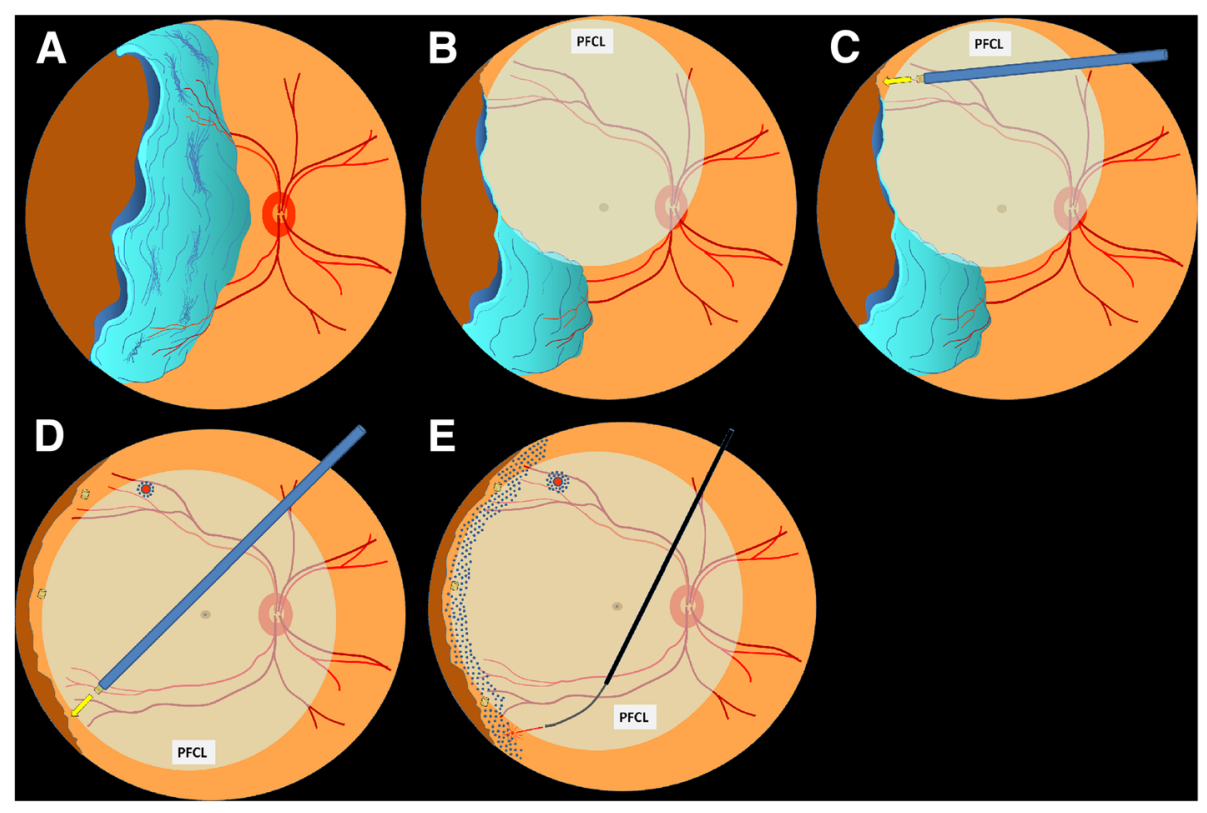

Fig. 2 a. The temporal side of retina was noted to have a rolled and stiffened edge at the posterior aspect of the giant retinal tear. b. Under 23gauge pars plana vitrectomy, the vitreous was removed and tractions were relieved as much as possible, then perfluorocarbon liquids (PFCL) was injected to help unfolding the large inverted retinal flap. $\mathbf{c}$ and $\mathbf{d}$. Three stainless steel retinal tacks were inserted along the posterior edge of the giant retinal tear using a modified Southerland intraocular forceps to help fixating the large inverted retinal flap. e. Fluid-gas exchange, endolaser photocoagulation and intraocular silicone oil tamponade were performed 
retinal tacks were inserted along the posterior edge of the giant retinal tear using a modified Southerland intraocular forceps [6] to help fixating the large inverted retinal flap (Fig. 2c and d). Then, fluid-gas exchange, endolaser photocoagulation and intraocular silicone oil tamponade were performed as well (Fig. 2e). Initial reattachment of his right retina was achieved and his best corrected visual acuity improved to 0.3 of his right eye postoperatively. There was no recurrent retinal detachment during follow up period of 19 months (Figs. $1 \mathrm{~b}$ and $\mathrm{c}$ ).

\section{Discussion}

Previous studies had reported that primary success rate for GRT treated by 20-gauge PPV using PFCL assistant without scleral buckling can achieve over $90 \%$ [7]. Another case series reported by Kunikata also suggested similar results for the primary success rate of managing GRT via MIVS [4]. However, treating patients with a rigid inverted retinal flap is relative difficult and retinal tack might make the surgical procedure easier. To date, there's no similar reports published in Taiwan currently. We performed MIVS for a case of GRT with retinal detachment. To remove the peripheral vitreous completely and release all possible vitreous traction without causing more damage to the retina, we use WAVs and high cutting rate vitrectomy system. Besides, for GRT with large inverted and rigid flap, we use retinal tacks to stabilize the flap in prevention of intraoperative or postoperative retinal slippage. In present case, reattachment was obtained after initial MIVS. The postoperative complication of cataract formation has been developed in this case and no other complications such as subretinal perfluorocarbon, retinal slippage, recurrent retinal detachment, proliferative vitreoretinopathy formation or macular pucker needing additional surgery during follow up period of 19 months. Reviewing the literatures, Ando et al. introduced polyacetal retinal tacks together with conventional 20G vitrectomy for GRT with $81 \%$ of patients with retinal total or partial attached. However, MIVS has the advantage of smaller sclerotomy which might reduce the possibility of occurrence of intraoperative iatrogenic retinal breaks, enhance patient's comfort and postoperative wound recovery. In addition, WAVs can improve the intraoperative field of view and offer better visibility and sharpness image of peripheral retina [5]. Currently, retinal tack had been abandoned by many surgeons due to unavailability and the concern of safety, we found it might be irreplaceable in cases of GRT with large inverted and rigid flap [8] and it may cause minimal or no retinal toxicity during as long as 21 years of follow-up [9]. To our knowledge, this is the first report of managing GRT with MIVS and retinal tacks in Taiwan.

\section{Conclusion}

Although our report has limitation of small case number and relatively short follow-up period, our findings indicate that MIVS is a safe, effective and feasible method for managing GRT. In addition, in cases with large, inverted and rigid retinal flap, retinal tacks for stabilizing retinal flap might be inevitable to prevent retinal redetachment.

\section{Abbreviations}

GRT: Giant retinal tear; MIVS: Microincisional vitrectomy surgery; PFCL: Perfluorocarbon liquids; WAVs: Wide-angle viewing systems

\section{Acknowledgements}

None.

Funding

No funding was obtained for this study.

Availability of data and materials

All the data supporting the conclusions of this article is included in the present article.

\section{Authors' contributions}

YCC was responsible for obtaining consent, collecting data and drafting the manuscript. TEK has helped preparing figures and manuscript drafting. LYC and TAC have helped manuscript and figures drafting. WHC has collected data and reviewed the literature. WCW has designed the overall concept, and revised the draft. All authors approved the final version.

Ethics approval and consent to participate

The local ethics committee ruled that no formal ethics approval was required in this case report.

The authors declare that they adhered to the CARE guidelines/methodology.

\section{Consent for publication}

Written informed consent was obtained from the patient for publication of this case report and any accompanying images.

\section{Competing interests}

The authors declare that they have no competing interests.

Conflicts of Interest No conflicting relationships exist for any of the authors.

\section{Publisher's Note}

Springer Nature remains neutral with regard to jurisdictional claims in published maps and institutional affiliations.

Received: 6 January 2018 Accepted: 12 October 2018

Published online: 22 October 2018

\section{References}

1. Shunmugam M, Ang GS, Lois N. Giant retinal tears. Surv Ophthalmol. 2014; 59:192-216.

2. Ando F, Kondo J. A plastic tack for the treatment of retinal detachment with giant tear. Am J Ophthalmol. 1983;95:260-1.

3. de Juan E Jr, Hickingbotham D, Machemer R. Retinal tacks. Am J Ophthalmol, 1985: 99: 272-274.

4. Kunikata H, Abe T, Nishida K. Successful outcomes of 25- and 23-gauge vitrectomies for giant retinal tear detachments. Ophthalmic Surg Lasers Imaging. 2011;42:487-92.

5. Inoue M. Wide-angle viewing system. Dev Ophthalmol. 2014:54:87-91.

6. O'Grady GE, Parel JM, Lee W, et al. Hypodermic stainless steel tacks and companion inserter designed for peripheral fixation of retina. Arch Ophthalmol. 1988;106:271-5. 
7. Chang S, Lincoff H, Zimmerman NJ, Fuchs W. Giant retinal tears: surgical techniques and results using perfluorocarbon liquids. Arch Ophthalmol. 1989;107:761-6.

8. Puustjärvi TJ, Teräsvirta ME. Retinal fixation of traumatic retinal detachment with metallic tacks: a case report with 10 years' follow-up. Retina. 2011;21:54-6.

9. Javey G1, Schwartz SG, Flynn HW Jr, Lee WG, Parel JM.Lack of toxicity of stainless steel retinal tacks during 21 years of follow-up. Ophthalmic Surg Lasers Imaging, 2009; 40: 75-76.

Ready to submit your research? Choose BMC and benefit from:

- fast, convenient online submission

- thorough peer review by experienced researchers in your field

- rapid publication on acceptance

- support for research data, including large and complex data types

- gold Open Access which fosters wider collaboration and increased citations

- maximum visibility for your research: over $100 \mathrm{M}$ website views per year

At $\mathrm{BMC}$, research is always in progress.

Learn more biomedcentral.com/submissions 